\title{
The Interval-Valued Choquet Integral Based on Admissible Permutations
}

\author{
Daniel Paternain, Laura De Miguel, Gustavo Ochoa, Inmaculada Lizasoain, Radko Mesiar, \\ and Humberto Bustince, Fellow, IEEE
}

\begin{abstract}
Aggregation or fusion of interval data is not a trivial task, since the necessity of arranging data arises in many aggregation functions, such as OWA operators or the Choquet integral. Some arranging procedures have been given to solve this problem, but they need certain parameters to be set. In order to solve this problem, in this work we propose the concept of an admissible permutation of intervals. Based on this concept, which avoids any parameter selection, we propose a new approach for the interval-valued Choquet integral that takes into account every possible permutation fitting to the considered ordinal structure of data. Finally, a consensus among all the permutations is constructed.
\end{abstract}

Index Terms-Information Fusion, Choquet Integral, IntervalValued Choquet Integral, Admissible Order, Admissible Permutation.

\section{INTRODUCTION}

A GGREGATION techniques are nowadays a very important tool, since the need to fuse several values (coming from different inputs) into a single one is a key step that appears in almost every application [1], [2], [3]. For example, in decision making problems, the inputs could be experts opinions or evaluations of criteria; in sensor fusing, data coming from different sensors must be combined so as to reduce uncertainty of data [4]; in image processing, the fusion of adjacent pixels or the fusion of pixels coming from different images is the foundation of image filtering, noise reconstruction, reduction, stereo vision, etc. [5], [6].

Extensions of fuzzy sets, such as interval-valued fuzzy sets, Atanassov's intuitionistic fuzzy sets, type-2 fuzzy sets, among others, have become very popular in many applications in order to deal with the uncertainty inherent to data [7], [8], [9], [10]. In this work we focus on intervals as the source of information that must be aggregated since, with moderate complexity, they allow to model uncertainty adequately. For example, information coming from sensors can be modeled by intervals when it takes inherent accuracy of instrumentation. In

Daniel Paternain, Laura De Miguel and Humberto Bustince are with the Departament of Statistics, Computer Science and Mathematics and with the Institute of Smart Cities, Universidad Publica de Navarra, 31006 Pamplona, Spain. e-mail: \{daniel.paternain,laura.demiguel,bustince\} @unavarra.es

G. Ochoa and I. Lizasoain are with the Departament of Statistics, Computer Science and Mathematics and with the Institute for Advances Materials INAMAT, Universidad Publica de Navarra, 31006 Pamplona, Spain. e-mail: \{ochoa,ilizasoain\}@unavarra.es

R. Mesiar is with the Department of Mathematics and Descriptive Geometry, Faculty of Civil Engineering, Slovak University of Technology, Bratislava, Slovakia and with the Institute for Research and Applications of Fuzzy Modelling, NSC IT4Innovations, University of Ostrava, Ostrava, Czech Republic

Manuscript received April 19, 2005; revised August 26, 2015. decision making, intervals are adequate when experts may not be able of assigning an exact opinion or numerical evaluation of an alternative. Moreover, an interval of labels, such as "something in between good and very good", could be more appropriate. In image processing, the intensity of a specific pixel may not be too informative. However, applications where the pixel intensity is transformed into an interval that take also into account neighbors' information have been proven to obtain better results in some applications [11], [12].

In order to extend aggregation functions such as the Choquet integral or OWA operators to the interval setting, one of the main difficulties is the arrangement of input data in a decreasing (or in an increasing) way. Genuine partial ordering of intervals excludes the possibility of a direct use of formulas for the (discrete) Choquet integral, where the weights of single inputs are derived from a considered measure by means of some permutation relevant to the ordinal structure of the real data to be aggregated. One way to solve this problem is related to Auman's approach to integration of set-valued functions [13]. This approach neglects the ordinal structure of the considered interval data, and, instead of, it deals separately with lower and upper bounds. An alternative approach, based on the concept of admissible order, was proposed in [14]. This approach performs the arrangement of data using a certain admissible order. Then, a new problem is opened - the choice of an appropriate admissible order. Our aim is to introduce a new method, based purely on the ordinal structure of interval data to be aggregated, thus preserving the original idea of Choquet [15].

To do so, in this paper we propose the concept of an admissible permutation of a set of intervals. This concept allows us to know how many different arrangements can be obtained by admissible orders. Then, we propose a new interval-valued Choquet integral that considers every possible arrangement (instead of single one) and constructs a consensus result among them.

Finally, we illustrate the use of admissible permutations in a decision-making problem based on interval preference relations.

This paper is organized as follows. In Section II we recall the main concepts used along this paper. In Section III we explore and analyze previous approaches to the interval Choquet integral. In Sections IV and V we introduce the concept of admissible permutation and interval-valued Choquet integral based on admissible permutations, respectively. We finish with an illustrative example of decision-making in Section VI and conclusions in Section VII. 


\section{PRELIMINARIES}

We start recalling the concept of an aggregation function.

Definition 1: [16] Let $(L, \preceq)$ be a bounded partially ordered set with a least element $0_{L}$ and a greatest element $1_{L}$. A mapping $M: L^{n} \rightarrow L$ is an $n$-ary aggregation function if it satisfies the properties:

(i) $M\left(0_{L}, \ldots, 0_{L}\right)=0_{L}$ and $M\left(1_{L}, \ldots, 1_{L}\right)=1_{L}$;

(ii) it is increasing in each argument, i.e., for all $\left(x_{1}, \ldots, x_{n}\right),\left(y_{1}, \ldots, y_{n}\right) \in L^{n}, M\left(x_{1}, \ldots, x_{n}\right) \preceq$ $M\left(y_{1}, \ldots, y_{n}\right)$ whenever $x_{1} \preceq y_{1}, \ldots, x_{n} \preceq y_{n}$.

Observe that if $L$ is the unit interval equipped with the standard ordering of reals, $L=[0,1]$, then we obtain the usual definition of aggregation function [1], [2], [3].

In this paper we deal with aggregation of intervals. Therefore, we take $L=L([0,1])$ as the set of all closed subintervals in $[0,1]$ :

$$
L([0,1])=\{\mathbf{x}=[\underline{x}, \bar{x}] \mid 0 \leq \underline{x} \leq \bar{x} \leq 1\} .
$$

Note that $L([0,1])$ is a partially ordered set with respect to the order relation $\leq_{L}$ defined in the following way: for any $\mathbf{x}, \mathbf{y} \in L([0,1])$,

$$
\mathbf{x} \leq_{L} \mathbf{y} \text { if and only if } \underline{x} \leq \underline{y} \text { and } \bar{x} \leq \bar{y} .
$$

In fact, $\left(L([0,1]), \leq_{L}\right)$ is a complete lattice where the least element is $0_{L}=[0,0]$ and the greatest element is $1_{L}=[1,1]$ ([17]). In this lattice, the infimum and supremum of any two elements are given, respectively, by

$$
\begin{aligned}
\mathbf{x}_{1} \wedge \mathbf{x}_{2} & =\left[\min \left(\underline{x}_{1}, \underline{x}_{2}\right), \min \left(\bar{x}_{1}, \bar{x}_{2}\right)\right] \\
\mathbf{x}_{1} \vee \mathbf{x}_{2} & =\left[\max \left(\underline{x}_{1}, \underline{x}_{2}\right), \max \left(\bar{x}_{1}, \bar{x}_{2}\right)\right] .
\end{aligned}
$$

Example 1: The following are examples of aggregation functions defined on $\left(L([0,1]), \leq_{L}\right)$ :

$$
\begin{aligned}
& \mathbf{M}_{\text {arith }}\left(\mathbf{x}_{1}, \ldots, \mathbf{x}_{n}\right)=\left[\frac{1}{n} \sum_{i=1}^{n} \underline{x}_{i}, \frac{1}{n} \sum_{i=1}^{n} \bar{x}_{i}\right] ; \\
& \mathbf{M}_{\min }\left(\mathbf{x}_{1}, \ldots, \mathbf{x}_{n}\right)=\left[\min _{i=1, \ldots, n} \underline{x}_{i}, \min _{i=1, \ldots, n} \bar{x}_{i}\right] ; \\
& \mathbf{M}_{\text {max }}\left(\mathbf{x}_{1}, \ldots, \mathbf{x}_{n}\right)=\left[\max _{i=1, \ldots, n} \underline{x}_{i}, \max _{i=1, \ldots, n} \bar{x}_{i}\right] ; \\
& \mathbf{M}_{\text {geom,arith }}\left(\mathbf{x}_{1}, \ldots, \mathbf{x}_{n}\right)=\left[\prod_{i=1}^{n} \underline{x}_{i}, \sum_{i=1}^{n} \bar{x}_{i}\right] ;
\end{aligned}
$$

Remark 1: Observe that $\mathbf{M}_{\min }$ and $\mathbf{M}_{\max }$ coincide with infimum and supremum of $\mathbf{x}_{1}, \ldots, \mathbf{x}_{n}$, respectively.

\section{A. Admissible orders on $L([0,1])$}

The order $\leq_{L}$ defined for intervals in subsection II is a partial order on $L([0,1])$. This means that it is not always possible to compare or arrange two arbitrary intervals. However, several aggregation functions defined on $[0,1]$, such as OWA operators or the Choquet and Sugeno integrals, are based on the arrangement of the inputs. Therefore, if we want to extend these aggregation functions to $L([0,1])$, we need to consider the problem associated with the partial order.
In order to solve the problem, in [18] the concept of admissible order was introduced. Admissible orders are, in fact, linear orders on $L([0,1])$ refining the partial order $\leq_{L}$.

Definition 2: [18] Consider the partially order set $(L([0,1]), \preceq)$. The order $\preceq$ on $L([0,1])$ is called an admissible order if

(i) $\preceq$ is a linear order on $L([0,1])$;

(ii) for all $\mathbf{x}, \mathbf{y} \in L([0,1]), \mathbf{x} \preceq \mathbf{y}$ whenever $\mathbf{x} \leq_{L} \mathbf{y}$.

Example 2: The following are examples of admissible orders:

(i) $\mathbf{x} \preceq_{\text {Lex } 1} \mathbf{y}$ (usual lexicographic order in $\mathbb{R}^{2}$ transformed onto $L([0,1]))$ if and only if $\underline{x}<\underline{y}$ or $(\underline{x}=\underline{y}$ and $\bar{x} \leq \bar{y})$;

(ii) $\mathbf{x} \preceq_{L e x 2} \mathbf{y}$ if and only if $\bar{x}<\bar{y}$ or $(\bar{x}=\bar{y}$ and $\underline{x} \leq y)$;

(iii) $\mathbf{x} \preceq_{X Y} \mathbf{y}$ (Xu-Yager order given in [19]) if and only if $\underline{x}+\bar{x}<\underline{y}+\bar{y}$ or $(\underline{x}+\bar{x}=\underline{y}+\bar{y}$ and $\bar{y}-\underline{y} \leq \bar{x}-\underline{x})$.

Admissible orders can be generated by means of two aggregation functions on $[0,1]$ fulfilling certain conditions. Let $K([0,1])=\left\{(\underline{x}, \bar{x}) \in[0,1]^{2} \mid \underline{x} \leq \bar{x}\right\}$.

Proposition 1: [18] Let $A, B:[0,1]^{2} \rightarrow[0,1]$ be two aggregation functions on $[0,1]$ such that, for all $(x, y),(u, v) \in$ $K([0,1])$, the equalities $A(x, y)=A(u, v)$ and $B(x, y)=$ $B(u, v)$ can hold simultaneously only if $(x, y)=(u, v)$. Define the relation $\preceq_{\mathrm{A}, \mathrm{B}}$ on $L([0,1])$ by $\mathbf{x} \preceq_{\mathrm{A}, \mathrm{B}} \mathbf{y}$ if and only if

$$
\begin{aligned}
& A(\underline{x}, \bar{x}) \quad<\quad A(\underline{y}, \bar{y}) \quad \text { or } \\
&(A(\underline{x}, \bar{x})=A(\underline{y}, \bar{y}) \quad \text { and } \quad B(\underline{x}, \bar{x}) \leq B(\underline{y}, \bar{y})) .
\end{aligned}
$$

Then, $\preceq_{\mathrm{A}, \mathrm{B}}$ is an admissible order on $L([0,1])$.

\section{B. Fuzzy measures and discrete Choquet integral}

Prior to the definition of the Choquet integral, we recall the concept of a fuzzy measure (see [20], [21]).

Definition 3: Let $X=\{1, \ldots, n\}$. A fuzzy measure over $X$ is a mapping $m: 2^{X} \rightarrow[0,1]$ such that

(i) $m(\emptyset)=0$ and $m(X)=1$;

(ii) If $E \subset F$, then $m(E) \leq m(F)$.

\section{Example 3:}

- The bottom fuzzy measure is defined by

$$
m_{*}(E)= \begin{cases}1 & \text { if } E=X ; \\ 0 & \text { otherwise }\end{cases}
$$

Observe that for any other fuzzy measure $m$ over $X$, it holds that $m_{*}(E) \leq m(E)$, for every $E \subseteq X$.

- The top fuzzy measure is defined by

$$
m^{*}(E)= \begin{cases}0 & \text { if } E=\emptyset \\ 1 & \text { otherwise }\end{cases}
$$

Observe that for any other fuzzy measure $m$ over $X$, it holds that $m(E) \leq m^{*}(E)$ for every $E \subseteq X$.

Definition 4: [15] Let $m: 2^{X} \rightarrow[0,1]$ be a fuzzy measure. The discrete Choquet integral of $x_{1}, \ldots, x_{n} \in[0,1]$ with respect to $m$ is defined by

$$
\begin{aligned}
C_{m}\left(x_{1}, \ldots, x_{n}\right)= & \sum_{i=1}^{n} x_{\sigma(i)}(m(\{\sigma(i), \ldots, \sigma(n)\})- \\
& m(\{\sigma(i+1), \ldots, \sigma(n)\}))
\end{aligned}
$$


where $\sigma:\{1, \ldots, n\} \rightarrow\{1, \ldots, n\}$ is a permutation such that $x_{\sigma(1)} \leq \cdots \leq x_{\sigma(n)}$ and $\left\{x_{\sigma(n+1)}, x_{\sigma(n)}\right\}=\emptyset$, by convention.

An alternative definition of the discrete Choquet integral was shown in [22], namely

$C_{m}\left(x_{1}, \ldots, x_{n}\right)=\sup \left\{\sum_{i=1}^{n} r_{i} m\left(E_{i}\right) \mid\left(r_{1}, \ldots, r_{n}\right) \in[0,1]^{n}\right.$,

$\left(E_{i}\right)_{i=1}^{n}$ is a chain in $X$ and $\left.\sum_{i=1}^{n} r_{i} 1_{E_{i}} \leq\left(x_{1}, \ldots, x_{n}\right)\right\}$.

where $1_{E_{i}}$ is the characteristic function of the set $E_{i}$. Note that the supremum is attained, i.e. it can be replaced by the max operator.

\section{DIFFERENT APPROACHES TO THE INTERVAL-VALUED CHOQUET INTEGRAL}

In the literature we find several approaches that extend the discrete Choquet integral to the interval setting. In this section we recall some of them and we show some new results. Both the advantages and disadvantages of each approach are analyzed.

\section{A. Interval-valued Choquet integral based on Auman's ap- proach and integral decomposition approach}

The first approach of interval-valued Choquet integral is based on Aumann's integral definition for set-valued functions [13].

Definition 5: Let $m: 2^{X} \rightarrow[0,1]$ be a fuzzy measure and let $\mathbf{x}_{1}, \ldots, \mathbf{x}_{n} \in L([0,1])$. The discrete interval-valued Choquet integral $\mathbf{C}_{m}\left(\mathbf{x}_{1}, \ldots, \mathbf{x}_{n}\right)$ with respect to $m$ is given by

$$
\mathbf{C}_{m}\left(\mathbf{x}_{1}, \ldots, \mathbf{x}_{n}\right)=\left\{C_{m}\left(x_{1}, \ldots, x_{n}\right) \mid x_{i} \in \mathbf{x}_{i}\right\} .
$$

Taking into account the monotonicity and continuity of the discrete Choquet integral, it follows that

$$
\mathbf{C}_{m}\left(\mathbf{x}_{1}, \ldots, \mathbf{x}_{n}\right)=\left[C_{m}\left(\underline{x}_{1}, \ldots, \underline{x}_{n}\right), C_{m}\left(\bar{x}_{1}, \ldots, \bar{x}_{n}\right)\right] .
$$

This means that the interval-valued Choquet integral based on Aumann's approach is given by the set of all Choquet integrals (defined on $[0,1]$ ) applied to every possible $n$-tuple of real numbers each of them within the corresponding interval.

Remark 2: Notice that $\mathbf{C}_{m_{*}}=\mathbf{M}_{\min }$ and $\mathbf{C}_{m^{*}}=\mathbf{M}_{\max }$.

Another approach to the discrete interval-valued Choquet integral is studied in [23], [22] as a particular case of decomposition integral, in which the set system is a chain of sets. We extend this approach to the interval setting $L([0,1])$ and we prove that, even though both approaches have different inspiration, they yield the same result.

Definition 6: Let $m: 2^{X} \rightarrow[0,1]$ be a fuzzy measure and let $\mathbf{x}_{1}, \ldots, \mathbf{x}_{n} \in L([0,1])$. The interval-valued Choquet integral $\mathbf{I}_{m}\left(\mathbf{x}_{1}, \ldots, \mathbf{x}_{n}\right)$ based on the decomposition integral is given by

$\mathbf{I}_{m}\left(\mathbf{x}_{1}, \ldots, \mathbf{x}_{n}\right)=\sup \left\{\sum_{i=1}^{n} \mathbf{r}_{i} m\left(E_{i}\right) \mid\left(\mathbf{r}_{1}, \ldots, \mathbf{r}_{n}\right) \in L([0,1])^{n}\right.$

$\left(E_{i}\right)_{i=1}^{n}$ is a chain in $2^{X}$ and $\left.\sum_{i=1}^{n} \mathbf{r}_{i} 1_{E_{i}} \leq_{L}\left(\mathbf{x}_{1}, \ldots, \mathbf{x}_{n}\right)\right\}$ where $1_{E_{i}}$ is the characteristic function of the set $E_{i}$.

Proposition 2: Let $m: 2^{X} \rightarrow[0,1]$ be a fuzzy measure. Then it holds that for any $\mathbf{x}_{1}, \ldots, \mathbf{x}_{n} \in L([0,1])$

$$
\mathbf{C}_{m}\left(\mathbf{x}_{1}, \ldots, \mathbf{x}_{n}\right)=\mathbf{I}_{m}\left(\mathbf{x}_{1}, \ldots, \mathbf{x}_{n}\right)
$$

Proof: Consider any situation in which $\sum_{i=1}^{n} \mathbf{r}_{i} 1_{E_{i}} \leq_{L}$ $\left(\mathbf{x}_{1}, \ldots, \mathbf{x}_{n}\right)$. Then, $\sum_{i=1}^{n} \underline{r}_{i} 1_{E_{i}} \leq\left(\underline{x}_{i}, \ldots, \underline{x}_{n}\right)$ and, due to Eq. 4, $\sum_{i=1}^{n} \underline{r}_{i} m\left(E_{i}\right) \leq C_{m}\left(\underline{x}_{i}, \ldots, \underline{x}_{n}\right)$. Thus, $\underline{I}_{m}\left(\mathbf{x}_{1}, \ldots, \mathbf{x}_{n}\right) \leq C_{m}\left(\underline{x}_{1}, \ldots, \underline{x}_{n}\right)$. Similarly, for the upper bounds we have that $I_{m}\left(\bar{x}_{1}, \ldots, \bar{x}_{n}\right) \leq C_{m}\left(\bar{x}_{1}, \ldots, \bar{x}_{n}\right)$ and hence

$$
\mathbf{I}_{m}\left(\mathbf{x}_{1}, \ldots, \mathbf{x}_{n}\right) \leq_{L} \mathbf{C}_{m}\left(\mathbf{x}_{1}, \ldots, \mathbf{x}_{n}\right)
$$

Based in Eqs. 3 and 4, as already mentioned at the end of previous section, it can be shown that there is a chain $\left(E_{i}\right)$ in $2^{X}$ and constants $c_{1}, \ldots, c_{n} \in[0,1]$ such that $\sum_{i=1}^{n} c_{i} 1_{E_{i}}=\left(\underline{x}_{1}, \ldots, \underline{x}_{n}\right)$ and $C_{m}\left(\underline{x}_{1}, \ldots, \underline{x}_{n}\right)=\sum_{i=1}^{n} c_{i} m\left(E_{i}\right)$. Putting $\mathbf{r}_{i}=\left[c_{i}, c_{i}\right]$, obviously $\sum_{i=1}^{n} \mathbf{r}_{i} 1_{E_{i}} \leq_{L} \quad\left(\mathbf{x}_{1}, \ldots, \mathbf{x}_{n}\right)$ and, thus, $C_{m}\left(\underline{x}_{1}, \ldots, \underline{x}_{n}\right) \leq \sum_{i=1}^{n} c_{i} m\left(E_{i}\right) \leq \underline{I}_{m}\left(\mathbf{x}_{1}, \ldots, \mathbf{x}_{n}\right)$. Similarly, one can show that $C_{m}\left(\bar{x}_{1}, \ldots, \bar{x}_{n}\right) \leq \bar{I}_{m}\left(\mathbf{x}_{1}, \ldots, \mathbf{x}_{n}\right)$ and hence

$$
\mathbf{C}_{m}\left(\mathbf{x}_{1}, \ldots, \mathbf{x}_{n}\right) \leq_{L} \mathbf{I}_{m}\left(\mathbf{x}_{1}, \ldots, \mathbf{x}_{n}\right)
$$

Summarizing, it holds that

$$
\mathbf{I}_{m}\left(\mathbf{x}_{1}, \ldots, \mathbf{x}_{n}\right)=\mathbf{C}_{m}\left(\mathbf{x}_{1}, \ldots, \mathbf{x}_{n}\right)
$$

Remark 3: Let us denote by

$$
\begin{aligned}
& A=\left\{\sum_{i=1}^{n} \mathbf{r}_{i} m\left(E_{i}\right) \mid\left(\mathbf{r}_{1}, \ldots, \mathbf{r}_{n}\right) \in L([0,1])^{n}\right. \\
& \left.\left(E_{i}\right)_{i=1}^{n} \text { is a chain in } 2^{X} \text { and } \sum_{i=1}^{n} \mathbf{r}_{i} 1_{E_{i}} \leq_{L}\left(\mathbf{x}_{1}, \ldots, \mathbf{x}_{n}\right)\right\}
\end{aligned}
$$

Notice that, in general $\sup \{A\} \notin A$.

Example 4: Let $\mathbf{x}_{1}=[0,1], \mathbf{x}_{2}=[1 / 2,1 / 2]$ and let $m: 2^{\{1,2\}} \rightarrow[0,1]$ be given by $m(\{1\})=a, m(\{2\})=b$, with $a, b \in[0,1]$. We have two possible chains, namely $E_{1}=\{1,2\}, E_{2}=\{1\}$ and $E_{1}=\{1,2\}, E_{2}=\{2\}$. For the first case we have that, according to the restriction $\mathbf{r}_{1} 1_{E_{1}}+\mathbf{r}_{2} 1_{E_{2}} \leq_{L}\left(\mathbf{x}_{1}, \mathbf{x}_{2}\right)$, we have that $\mathbf{r}_{1}+\mathbf{r}_{2} \leq_{L}[0,1]$ and $\mathbf{r}_{1} \leq_{L}[1 / 2,1 / 2]$, that implies $\underline{r}_{1}=\underline{r}_{2}=0, \bar{r}_{1} \leq 1 / 2$ and $\bar{r}_{1}+\bar{r}_{2} \leq 1$. Then, $\sup \left\{\mathbf{r}_{1} m\left(E_{1}\right)+\mathbf{r}_{2} m\left(E_{2}\right)\right\}=$ $\left[0, \underline{r}_{1}+a \underline{r}_{2}\right]=[0,(a+1) / 2]$ is achieved with $\bar{r}_{1}=\bar{r}_{2}=$ $1 / 2$. In the second case, we have that $\mathbf{r}_{1} \leq_{L}[0,1]$ and $\mathbf{r}_{1}+\mathbf{r}_{2} \leq_{L}[1 / 2,1 / 2]$, which implies $\underline{r}_{1}=0, \bar{r}_{1}+\bar{r}_{2} \leq 1 / 2$. Then, $\sup \left\{\mathbf{r}_{1} m\left(E_{1}\right)+\mathbf{r}_{2} m\left(E_{2}\right)\right\}=\left[\underline{r}_{1}+b \underline{r}_{2}, \bar{r}_{1}+b \bar{r}_{2}\right]=$ ,$[b / 2, b / 2]$ is achieved with $\mathbf{r}_{1}=[0,0], \mathbf{r}_{2}=[1 / 2,1 / 2]$. Finally, $\mathbf{I}_{m}\left(\mathbf{x}_{1}, \mathbf{x}_{2}\right)=[b / 2,(a+1) / 2]$. It is easy to see that whenever $b>0$, then $\sup \{A\} \notin A$.

Taking now Auman's approach, we have that $\mathbf{C}_{m}\left(\mathbf{x}_{1}, \mathbf{x}_{2}\right)=$ $\left[C_{m}(0,1 / 2), C_{m}(1,1 / 2)\right]=[b / 2,(a+1) / 2]$. 


\section{B. Interval-valued Choquet integrals induced by admissible orders}

The previous approach to the interval-valued Choquet integral did not consider the ordinal structure of data. In [14], a new approach to the interval-valued Choquet integral was given. The originality of this new approach is based on the arrangement of interval inputs using any admissible order. In this sense, each admissible order generates a specific intervalvalued Choquet integral.

Definition 7: Let $\mathbf{x}_{1}, \ldots, \mathbf{x}_{n} \in L([0,1])$ and let $m: 2^{X} \rightarrow$ $[0,1]$ be a fuzzy measure. The discrete interval-valued Choquet integral with respect to an admissible order $\preceq_{\mathrm{A}, \mathrm{B}}$ with notation $\mathbf{C}_{m}^{-\mathrm{A}, \mathrm{B}}$ is given by

$$
\begin{aligned}
& \mathbf{C}_{m}^{\prec \mathrm{A}, \mathrm{B}}\left(\mathbf{x}_{1}, \ldots, \mathbf{x}_{n}\right)=\sum_{i=1}^{n} \mathbf{x}_{\sigma_{A, B}(i)}\left(m \left(\left\{\sigma_{A, B}(i), \ldots,\right.\right.\right. \\
& \left.\left.\left.\sigma_{A, B}(n)\right\}\right)-m\left(\left\{\sigma_{A, B}(i+1), \ldots, \sigma_{A, B}(n)\right\}\right)\right)
\end{aligned}
$$

where $\sigma_{A, B}:\{1, \ldots, n\} \rightarrow\{1, \ldots, n\}$ is a permutation such that

$$
\mathbf{x}_{\sigma_{A, B}(1)} \preceq_{\mathrm{A}, \mathrm{B}} \cdots \preceq_{\mathrm{A}, \mathrm{B}} \mathbf{x}_{\sigma_{A, B}(n)}
$$

and $\left\{\sigma_{A, B}(n), \sigma_{A, B}(n+1)\right\}=\emptyset$ by convention.

One of the main advantages of this approach is the fact that $\mathbf{C}_{m}^{-\mathrm{A}, \mathrm{B}}$ generalizes the usual discrete Choquet integral defined on $[0,1]$, since

$$
\begin{gathered}
\mathbf{C}_{m}^{\prec \mathrm{A}, \mathrm{B}}\left(\left[x_{1}, x_{1}\right], \ldots,\left[x_{n}, x_{n}\right]\right)= \\
{\left[C_{m}\left(x_{1}, \ldots, x_{n}\right), C_{m}\left(x_{1}, \ldots, x_{n}\right)\right] .}
\end{gathered}
$$

Besides, if every input is comparable with respect to the partial order $\leq_{L}$, it obtains the same result than Aumann's approach, as it is proven in [14].

Moreover, comparing this approach with Auman's, we notice that interval inputs are considered as a whole, and lower/upper bounds are not split in the calculation of the integral. This means that the ordinal structure of data, which is determined by the admissible order, is taken into account for obtaining the fused value. This fact makes the interval-valued Choquet integral with respect to an admissible order more adequate than other approaches in problems such as multi-criteria decision-making. In this kind of problems, there usually exists a close relation between the fuzzy measure (that may represent interaction between criteria) and the interval inputs (that may represent the degree of satisfaction of criteria).

On the other side, some drawbacks of this proposal arise. First, the number of admissible orders is infinite and many of them are equivalent. Second, but related, it is very difficult to know which order must be used for an specific application.

In the following two sections we solve these problems using the concept of admissible permutations and defining an interval-valued Choquet integral based on every admissible permutation.

\section{THE CONCEPT OF ADMISSIBLE PERMUTATION AND ITS RELATION WITH ADMISSIBLE ORDERS}

As we have mentioned in the previous section, there exist infinitely many admissible orders. In fact, as it is proven in Proposition 3.8 of [18], many admissible orders are equivalent and yield in the same arrangement for a fixed vector of elements. Moreover, when we order a finite number of inputs (intervals), the number of possible arrangements is again finite, even if the number of admissible orders is infinite.

It is also important to mention that, given $n$ intervals, the total $n$ ! of its potential permutations need not be allowed. This is due to the fact that admissible orders are refinements of the usual partial order $\leq_{L}$. Therefore, given $\mathbf{x}_{1}, \ldots, \mathbf{x}_{n} \in$ $L([0,1])$ and a permutation $\sigma:\{1, \ldots, n\} \rightarrow\{1, \ldots, n\}$, if $\mathbf{x}_{i}<_{L} \quad \mathbf{x}_{j}$, then we should ensure that $\sigma^{-1}(i)<\sigma^{-1}(j)$. Moreover, if $\mathbf{x}_{i}=\mathbf{x}_{j}$, then for any $k$ between $\sigma^{-1}(i)$ and $\sigma^{-1}(j)$, we should ensure that $\mathbf{x}_{i}=\mathbf{x}_{\sigma(k)}=\mathbf{x}_{j}$. In order to clarify this idea, we introduce the concept of an admissible permutation of data.

Definition 8: Let $\mathbf{x}_{1}, \ldots, \mathbf{x}_{n} \in L([0,1])$. A permutation $\sigma:\{1, \ldots, n\} \rightarrow\{1, \ldots, n\}$ of $\mathbf{x}_{1}, \ldots, \mathbf{x}_{n}$ is said to be an admissible permutation with respect to the partial order $\leq_{L}$ if

(i) for every $\mathbf{x}_{i}<_{L} \mathbf{x}_{j}$, we have that $\sigma^{-1}(i)<\sigma^{-1}(j)$ and

(ii) for each $\mathbf{x}_{i}$, the set $\left\{\sigma^{-1}(j) \mid j \in\{1, \ldots, n\}\right.$ with $\mathbf{x}_{i}=$ $\left.\mathbf{x}_{j}\right\}$ is an interval in $\mathbb{N}$.

The first property of Definition 8 ensures that the permutation does not alter the order of comparable intervals with the partial order. The second property ensures that equal intervals are ordered consecutively.

Example 5: Consider the following set of inputs: $\mathbf{x}_{1}=[0,0]$, $\mathbf{x}_{2}=[0.1,0.3], \mathbf{x}_{3}=[0.15,0.25], \mathbf{x}_{4}=[0.2,0.2], \mathbf{x}_{5}=$ $[0.2,0.4], \mathbf{x}_{6}=[0.3,0.7], \mathbf{x}_{7}=[0.4,0.5]$ and $\mathbf{x}_{8}=[0.6,0.8]$. We show the Hasse diagram of these intervals in the lattice $\left(L([0,1]), \leq_{L}\right)$.

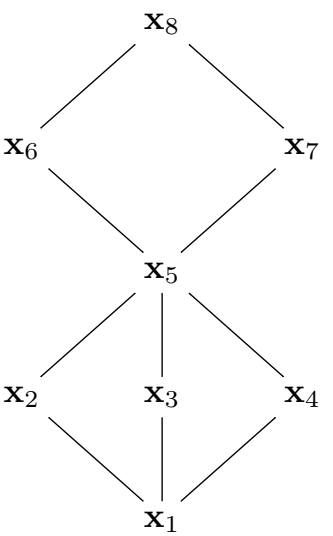

It is easy to see that there exist 12 admissible permutations of these intervals. Since $\mathbf{x}_{2}, \mathbf{x}_{3}$ and $\mathbf{x}_{4}$ are incomparable, there exist $3 !=6$ permutations of $\{2,3,4\}$. Now, since $\mathbf{x}_{6}$ and $\mathbf{x}_{7}$ are also incomparable, we have $2 !=2$ permutations of $\{6,7\}$. Finally, the number of admissible permutations is given by $3 ! 2 !=12$, drastically reducing the number $8 !=40320$ of all possible permutations.

As we have mentioned, many different admissible orders may induce the same arrangement of a set of intervals. Moreover, it is not easy to know a priori which admissible order should be used in order to obtain a certain arrangement. For these reasons, the importance of admissible permutations lies in the fact that, for a particular input vector, we can omit the choice of the admissible order. This is proven in the next 
theorem, in which we prove that any admissible permutation is induced by a certain admissible order.

Theorem 1: Let $\mathbf{x}_{1}, \ldots, \mathbf{x}_{n} \in L([0,1])$. The permutation $\sigma:\{1, \ldots, n\} \rightarrow\{1, \ldots, n\}$ is an admissible permutation of $\mathbf{x}_{1}, \ldots, \mathbf{x}_{n}$ if and only if there exists an admissible order $\preceq_{\mathrm{A}, \mathrm{B}}$ such that

$$
\mathbf{x}_{\sigma(1)} \preceq_{\mathrm{A}, \mathrm{B}} \cdots \preceq_{\mathrm{A}, \mathrm{B}} \mathbf{x}_{\sigma(n)} \cdot
$$

Proof: Sufficiency is direct, since $\preceq_{\mathrm{A}, \mathrm{B}}$ refines the partial order $\leq_{L}$. To prove necessity, we must find a pair of admissible aggregation functions $(A, B)$. Let us start by constructing, for each $\mathbf{x}_{i}$, the set $E_{i}=\left\{j \in\{1, \ldots, n\} \mid \mathbf{x}_{i}=\mathbf{x}_{j}\right\}$. Now, define $\tilde{A}(0,0)=0, \tilde{A}(1,1)=1$ and, for each $\mathbf{x}_{i} \notin\{[0,0],[1,1]\}$,

$$
\tilde{A}\left(\underline{x}_{i}, \bar{x}_{i}\right)=\frac{\min \left\{\sigma^{-1}(j) \mid j \in E_{i}\right\}}{n+1} .
$$

Observe that, for a function $\tilde{A}:[0,1]^{2} \rightarrow[0,1]$, the values $\tilde{A}\left(\underline{x}_{i}, \bar{x}_{i}\right)$ for every $i \in\{1, \ldots, n\}, \tilde{A}(0,0)$ and $\tilde{A}(1,1)$ are compatible with the constraints for aggregation functions, i.e. boundary conditions and monotonicity. Thus, we can extend $\tilde{A}$ to the whole domain $[0,1]^{2}$, for every $(u, v) \in[0,1]^{2}$, by taking

$$
\tilde{A}(u, v)= \begin{cases}\min \left\{\tilde{A}\left(\underline{x}_{i}, \bar{x}_{i}\right) \mid i \in E_{(u, v)}\right\} & \text { if } E_{(u, v)} \neq \emptyset \\ 1 & \text { otherwise }\end{cases}
$$

where $E_{(u, v)}=\left\{j \in\{1, \ldots, n\} \mid(u, v) \leq\left(\underline{x}_{j}, \bar{x}_{j}\right)\right\}$. Now we have to prove the monotonicity of $\tilde{A}$ in the whole domain. Take arbitrary $u, u^{\prime}, v, v^{\prime} \in[0,1]$ with $u \leq u^{\prime}$ and $v \leq v^{\prime}$. If $E_{\left(u^{\prime}, v^{\prime}\right)}=\emptyset$ then $\tilde{A}(u, v) \leq \tilde{A}\left(u^{\prime}, v^{\prime}\right)$. Otherwise, whenever $i \in E_{\left(u^{\prime}, v^{\prime}\right)}$ it holds that $i \in E_{(u, v)}$ since $u \leq u^{\prime} \leq \underline{x}_{i}$ and $v \leq v^{\prime} \leq \bar{x}_{i}$. However, the contrary is not true, so $E_{\left(u^{\prime}, v^{\prime}\right)} \subseteq$ $E_{(u, v)}$ and, therefore, $\tilde{A}(u, v) \leq \tilde{A}\left(u^{\prime}, v^{\prime}\right)$. Finally, define the mappings $A, B:[0,1]^{2} \rightarrow[0,1]$ in the following way:

$$
\begin{aligned}
A(x, y) & =\frac{\tilde{A}(x, y)+\frac{x y}{n+1}}{1+\frac{1}{n+1}} \\
B(x, y) & =\frac{x^{2}+y}{2} .
\end{aligned}
$$

It is clear that $A, B$ are aggregation functions, due to the increasing monotonicity of $\widetilde{A}$. Now, let us prove that they form an admissible pair of aggregation functions. Suppose any $(x, y),(u, v) \in K([0,1])$ such that $A(x, y)=A(u, v)$ and suppose that $\tilde{A}(x, y)<\tilde{A}(u, v)$. Having in mind the construction method of $\tilde{A}$, we have that $\tilde{A}(u, v)-\tilde{A}(x, y) \geq$ $\frac{1}{n+1}$, so it implies that $x y \geq 1+u v$. But this is only possible if $x=y=1$ and we have a contradiction since $\tilde{A}(x, y)=1<\tilde{A}(u, v)$. The same reasoning can be done with the supposition $\tilde{A}(u, v)<\tilde{A}(x, y)$ and, therefore, the assumption $A(x, y)=A(u, v)$ implies that $\tilde{A}(x, y)=\tilde{A}(u, v)$ and, therefore, $x y=u v$. Now, if we consider the second equality $B(x, y)=B(u, v)$, we have that $x^{2}+y=u^{2}+v$. It is only a matter of calculation to prove that, if $x y=u v$ and $x^{2}+y=u^{2}+v$ hold simultaneously, then necessarily $x=u$ and $y=v$, so the pair $(A, B)$ is admissible and $\preceq_{\mathrm{A}, \mathrm{B}}$ is an admissible order. Moreover, it satisfies

$$
\mathbf{x}_{\sigma(1)} \preceq_{\mathrm{A}, \mathrm{B}} \cdots \preceq_{\mathrm{A}, \mathrm{B}} \mathbf{x}_{\sigma(n)}
$$

which proves the necessity of the theorem.

It is important to mention that both concepts, admissible order and admissible permutation, are not equivalent, since the definition of admissible permutation depends on each specific input vector. For a fixed input vector, Theorem 1 shows, on the one hand, that each admissible order induces an admissible permutation and, on the other hand, that each admissible permutation can be obtained by some admissible order. However, recall that we can have many admissible permutations of a given vector $\left(\mathbf{x}_{1}, \ldots, \mathbf{x}_{n}\right) \in L([0,1])^{n}$. For each admissible permutation $\sigma$, we can obtain a fused value $C_{m}^{\sigma}$ which is given by

$$
\begin{aligned}
\mathbf{C}_{m}^{\sigma}\left(\mathbf{x}_{1}, \ldots, \mathbf{x}_{n}\right)= & \sum_{i=1}^{n} \mathbf{x}_{\sigma(i)}(m(\{\sigma(i), \ldots, \sigma(n)\})- \\
& m(\{\sigma(i+1), \ldots, \sigma(n)\})) .
\end{aligned}
$$

Notice that, if $\left(\mathbf{x}_{1}, \ldots, \mathbf{x}_{n}\right) \in L([0,1])$ have $p$ admissible permutations $\sigma_{1}, \ldots, \sigma_{p}$, with $1 \leq p \leq n$ !, then we can obtain $p$ fused values $\mathbf{C}_{m}^{\sigma_{1}}, \ldots, \mathbf{C}_{m}^{\sigma_{p}}$.

\section{THE INTERVAL-VALUED CHOQUET INTEGRAL WITH RESPECT TO EVERY ADMISSIBLE PERMUTATION}

In the previous section we have proposed the concept of admissible permutation and we have seen that, given a set of intervals, there exists a finite number of admissible permutations (that may vary from 1 to $n$ !) for sorting them. Even though we have solved the problem of having infinitely many admissible orders, we still have a second problem: which admissible permutation should be used. In fact, given a vector $\left(\mathbf{x}_{1}, \ldots, \mathbf{x}_{n}\right) \in L([0,1])$, we can potentially obtain as many fused values as the number of admissible permutations of the input vector.

To solve this problem, our proposal consists in defining a new interval-valued Choquet integral whose result is given as a consensus or agreed value obtained considering each individual admissible permutation. We propose to obtain this consensus by calculating the arithmetic mean of each fused value $\mathbf{C}_{m}^{\sigma}$, as it is explained in the following definition.

Definition 9: Let $\mathbf{x}_{1}, \ldots, \mathbf{x}_{n} \in L([0,1])$ and let $m: 2^{n} \rightarrow$ $[0,1]$ be a fuzzy measure. Let $\sigma_{1}, \ldots, \sigma_{p}:\{1, \ldots, n\} \rightarrow$ $\{1, \ldots, n\}$ be the set of all admissible permutations of $\mathbf{x}_{1}, \ldots, \mathbf{x}_{n}$. The consensus (via arithmetic mean) intervalvalued Choquet integral $\mathbf{C}_{m}^{\text {arith }}$ with respect to $m$ is given, respectively, by

$$
\begin{aligned}
\mathbf{C}_{m}^{\text {arith }}\left(\mathbf{x}_{1}, \ldots, \mathbf{x}_{n}\right)=\mathbf{M}_{\text {arith }}\left(\mathbf{C}_{m}^{\sigma_{1}}\left(\mathbf{x}_{1}, \ldots, \mathbf{x}_{n}\right), \ldots,\right. \\
\left.\mathbf{C}_{m}^{\sigma_{p}}\left(\mathbf{x}_{1}, \ldots, \mathbf{x}_{n}\right)\right)
\end{aligned}
$$

Remark 4: Although in this paper we focus on the consensus interval-valued Choquet integral, it is interesting to explore the use of the extreme functions, such as the supremum and infimum operations instead of the arithmetic mean, namely $\mathbf{C}_{m}^{\text {inf }}, \mathbf{C}_{m}^{\text {sup }}: L([0,1])^{n} \rightarrow L([0,1])$ given by

$$
\begin{aligned}
\mathbf{C}_{m}^{\text {inf }}\left(\mathbf{x}_{1}, \ldots, \mathbf{x}_{n}\right)=\inf \left\{\mathbf{C}_{m}^{\sigma_{1}}\left(\mathbf{x}_{1}, \ldots, \mathbf{x}_{n}\right), \ldots,\right. \\
\\
\left.\mathbf{C}_{m}^{\sigma_{p}}\left(\mathbf{x}_{1}, \ldots, \mathbf{x}_{n}\right)\right\}, \\
\mathbf{C}_{m}^{\text {sup }}\left(\mathbf{x}_{1}, \ldots, \mathbf{x}_{n}\right)=\sup \left\{\mathbf{C}_{m}^{\sigma_{1}}\left(\mathbf{x}_{1}, \ldots, \mathbf{x}_{n}\right), \ldots,\right. \\
\left.\mathbf{C}_{m}^{\sigma_{p}}\left(\mathbf{x}_{1}, \ldots, \mathbf{x}_{n}\right)\right\} .
\end{aligned}
$$


In this sense, $\mathbf{C}_{m}^{i n f}$ can be considered as a pessimistic evaluation, while $\mathbf{C}_{m}^{\text {sup }}$ can be considered as an optimistic one. The choice of a decision maker between these possible aggregation methods depends either on his/her attitude or on the nature of the problem to be solved.

Example 6: Consider the following set of intervals that must be aggregated using the interval-valued Choquet integral:

$$
\begin{aligned}
& \mathbf{x}_{1}=[0.1,1], \\
& \mathbf{x}_{2}=[0.3,0.9], \\
& \mathbf{x}_{3}=[0.4,0.4] .
\end{aligned}
$$

Let $m$ be a fuzzy measure given by

$$
\begin{array}{lll}
m(\{\emptyset\})=0, & & \\
m(\{1\})=0.1, & m(\{2\})=0.5, & m(\{3\})=0.2, \\
m(\{1,2\})=0.5, & m(\{1,3\})=0.3, & m(\{2,3\})=0.9, \\
m(\{1,2,3\})=1 . & &
\end{array}
$$
by

First, recall that the result of Aumann's approach is given

$$
\begin{aligned}
\mathbf{C}_{m}\left(\mathbf{x}_{1}, \mathbf{x}_{2}, \mathbf{x}_{3}\right)= & {\left[C_{m}(0.1,0.3,0.4), C_{m}(1,0.9,0.4)\right]=} \\
& {[0.3,0.66] }
\end{aligned}
$$

Now, let us calculate the interval-valued Choquet integral with respect to every admissible permutation. Since $\mathbf{x}_{1}, \mathbf{x}_{2}, \mathbf{x}_{3}$ are not comparable (by means of $\leq_{L}$ ) there exist six admissible permutations $\sigma_{1}, \ldots, \sigma_{6}$, that are given in the following table

$$
\begin{aligned}
& \sigma_{1}=(1,2,3) \\
& \sigma_{2}=(1,3,2) \\
& \sigma_{3}=(2,1,3) \\
& \sigma_{4}=(2,3,1) \\
& \sigma_{5}=(3,1,2) \\
& \sigma_{6}=(3,2,1)
\end{aligned}
$$

Then, we have that

$$
\begin{aligned}
& \mathbf{C}_{m}^{\sigma_{1}}\left(\mathbf{x}_{1}, \mathbf{x}_{2}, \mathbf{x}_{3}\right)=[0.30,0.81] \\
& \mathbf{C}_{m}^{\sigma_{2}}\left(\mathbf{x}_{1}, \mathbf{x}_{2}, \mathbf{x}_{3}\right)=[0.32,0.71] \\
& \mathbf{C}_{m}^{\sigma_{3}}\left(\mathbf{x}_{1}, \mathbf{x}_{2}, \mathbf{x}_{3}\right)=[0.30,0.81] \\
& \mathbf{C}_{m}^{\sigma_{4}}\left(\mathbf{x}_{1}, \mathbf{x}_{2}, \mathbf{x}_{3}\right)=[0.30,0.81] \\
& \mathbf{C}_{m}^{\sigma_{5}}\left(\mathbf{x}_{1}, \mathbf{x}_{2}, \mathbf{x}_{3}\right)=[0.35,0.65] \\
& \mathbf{C}_{m}^{\sigma_{6}}\left(\mathbf{x}_{1}, \mathbf{x}_{2}, \mathbf{x}_{3}\right)=[0.33,0.66] .
\end{aligned}
$$

Finally, we can calculate the consensus interval-valued Choquet integral by using the arithmetic mean of the previous values

$$
\mathbf{C}_{m}^{\text {arith }}([0.1,1],[0.3,0.9],[0.4,0.4])=[0.317,0.742] .
$$

Observe that $\mathbf{C}_{m} \leq{ }_{L} \mathbf{C}_{m}^{\text {arith }}$ for this example and, specifically, the upper bounds are much more distant. This is probably explained by how Auman's approach uses two different permutations for the lower and upper bounds. In the case of the upperbounds, we have that $\bar{x}_{3}<\overline{x_{2}}<\overline{x_{1}}$ and, therefore,

$$
\begin{aligned}
\bar{C}_{m}= & \bar{x}_{3}(m(\{1,2,3\})-m(\{1,2\}))+ \\
& \bar{x}_{2}(m(\{1,2\})-m(\{1\}))+ \\
& \bar{x}_{1} m(\{1\}) .
\end{aligned}
$$

Since $(m(\{1,2,3\})-m(\{1,2\}))=0.5, \bar{x}_{3}$ gets more importance in the fused output, thus explaining the behavior of $\mathbf{C}_{m}$ in contrast with $\mathbf{C}_{m}^{\text {arith }}$.

Now, we prove that our proposed integral extends the standar Choquet integral by considering degenerated intervals (numbers).

Proposition 3: It holds that $\mathbf{C}_{m}^{\text {arith }}\left(\left[x_{1}, x_{1}\right], \ldots,\left[x_{n}, x_{n}\right]\right)=$ $\left[C_{m}\left(x_{1}, \ldots, x_{n}\right), C_{m}\left(x_{1}, \ldots, x_{n}\right)\right]$ for every $x_{1}, \ldots, x_{n} \in$ $[0,1]$.

Proof: Notice that if there is a unique admissible permutation, then the result is clear. Otherwise, observe that $\mathbf{C}_{m}^{\sigma_{1}}\left(\left[x_{1}, x_{1}\right], \ldots,\left[x_{n}, x_{n}\right]\right)=$ $\cdots \quad=\quad \mathbf{C}_{m}^{\sigma_{p}}\left(\left[x_{1}, x_{1}\right], \ldots,\left[x_{n}, x_{n}\right]\right)=$ $\left[C_{m}\left(x_{1}, \ldots, x_{n}\right), C_{m}\left(x_{1}, \ldots, x_{n}\right)\right]$, being $\sigma_{1}, \ldots, \sigma_{p}$ the set of admissible permutatinons of $\left(\left[x_{1}, x_{1}\right], \ldots,\left[x_{n}, x_{n}\right]\right)$ and the result follows.

The next proposition shows interesting behaviors of $\mathbf{C}_{m}^{\text {arith }}$ when certain input vectors are considered. The first result shows that if every interval considered can be compared by means of $\leq_{L}$, then we obtain Auman's approach. In the opposite way, if none of the intervals can be compared (they form an antichain), then no order structure can be obtained and the integral yields in the arithmetic mean.

Proposition 4: The following items hold:

(i) if $\mathbf{x}_{1}, \ldots, \mathbf{x}_{n}$ form a chain, then $\mathbf{C}_{m}^{\text {arith }}\left(\mathbf{x}_{1}, \ldots, \mathbf{x}_{n}\right)=$ $\mathbf{C}_{m}\left(\mathbf{x}_{1}, \ldots, \mathbf{x}_{n}\right)=\mathbf{C}_{m}^{\preceq A, B}$ for every admissible order $\preceq_{A, B}$.

(ii) if $\mathbf{x}_{1}, \ldots, \mathbf{x}_{n}$ form an antichain, then for any fuzzy measure $m, \mathbf{C}_{m}^{\text {arith }}\left(\mathbf{x}_{1}, \ldots, \mathbf{x}_{n}\right)=\mathbf{M}_{\text {arith }}\left(\mathbf{x}_{1}, \ldots, \mathbf{x}_{n}\right)$.

Proof: Direct.

We now investigate whether our new approach is an interval aggregation function and which properties of aggregation functions are satisfied. The next proposition shows that our approach is idempotent and bounded by the infimum and supremum of inputs, two main properties satisfied by averaging functions or means [2].

Proposition 5: The following items hold:

(i) $\mathbf{C}_{m}^{\text {arith }}(\mathbf{x}, \ldots, \mathbf{x})=\mathbf{x}$ for every $\mathbf{x} \in L([0,1])$;

(ii) $\inf \left\{\mathbf{x}_{1}, \ldots, \mathbf{x}_{n}\right\} \quad \leq_{L} \quad \mathbf{C}_{m}^{\text {arith }}\left(\mathbf{x}_{1}, \ldots, \mathbf{x}_{n}\right) \quad \leq_{L}$ $\sup \left\{\mathbf{x}_{1}, \ldots, \mathbf{x}_{n}\right\}$ for every $\mathbf{x}_{1}, \ldots, \mathbf{x}_{n} \in L([0,1])$;

Proof: $(i)$ Although there exist $n$ ! admissible permutations of $(\mathbf{x}, \ldots, \mathbf{x})$, we have that $\mathbf{C}_{m}^{\sigma_{1}}(\mathbf{x}, \ldots, \mathbf{x})=\cdots=$ $\mathbf{C}_{m}^{\sigma_{n !}}(\mathbf{x}, \ldots, \mathbf{x})=\mathbf{x}$, since $\mathbf{M}_{\text {arith }}$ is idempotent. (ii) Boundedness holds since $\mathbf{C}_{m}^{\sigma}$ and $\mathbf{M}_{\text {arith }}$ are also bounded by infimum and supremum of inputs.

However, we cannot consider $\mathbf{C}_{m}^{\text {arith }}$ as an interval aggregation functions since the monotonicity is not satisfied. This fact is herited from the same possible failure of $\mathbf{C}_{m}^{\prec A, B}$ integrals.

Example 7: Let $n=3$ and $\mathbf{x}_{1}=[0.2,0.9], \mathbf{x}_{2}=[0.3,0.7]$ and $\mathbf{x}_{3}=[0.5,0.6]$. Let $m=m_{*}$ (bottom fuzzy measure). Since $\mathbf{x}_{1}, \mathbf{x}_{2}, \mathbf{x}_{3}$ form an antichain, then $\mathbf{C}_{m}^{\text {arith }}\left(\mathbf{x}_{1}, \mathbf{x}_{2}, \mathbf{x}_{3}\right)=$ $\mathbf{M}_{\text {arith }}\left(\mathbf{x}_{1}, \mathbf{x}_{2}, \mathbf{x}_{3}\right)=\left[\frac{1}{3}, \frac{11}{15}\right]$. Suppose now that we increase $\mathbf{x}_{1}$ and $\mathbf{x}_{3}$ to $\mathbf{x}_{1}^{\prime}=\mathbf{x}_{3}^{\prime}=[1,1]$. Now, $\mathbf{x}_{1}^{\prime}, \mathbf{x}_{2}, \mathbf{x}_{3}^{\prime}$ form a chain and $\mathbf{C}_{m}^{\text {arith }}\left(\mathbf{x}_{1}^{\prime}, \mathbf{x}_{2}, \mathbf{x}_{3}^{\prime}\right)=\mathbf{C}_{m}\left(\mathbf{x}_{1}^{\prime}, \mathbf{x}_{2}, \mathbf{x}_{3}^{\prime}\right)=[0.3,0.7]$. Note that, while $\mathbf{x}_{1} \leq_{L} \quad \mathbf{x}_{1}^{\prime}$ and $\mathbf{x}_{3} \leq_{L} \quad \mathbf{x}_{3}^{\prime}$, we have that $[0.3,0.7] \leq_{L}\left[\frac{1}{3}, \frac{11}{15}\right]$ and monotonicity is not satisfied. 
Even if $\mathbf{C}_{m}^{\text {arith }}$ does not fulfill monotonicity, new trends in aggregation functions are introducing new types of monotonicities. This is the case of weak monotonicity [24] or directional monotonicity [25]. In fact, our new proposal can be seen as a weak monotone function or a directional monotone function with respect to $r=(1, \ldots, 1)$, since $\mathbf{C}_{m}^{\text {arith }}$ is invariant under translation.

Lemma 1: Let $\mathbf{x}_{1}, \ldots, \mathbf{x}_{n} \in L([0,1])$ and let $\mathbf{k}, \mathbf{r} \in$ $L([0,1])$ such that $\mathbf{k} \mathbf{x}_{1}+\mathbf{r}, \ldots, \mathbf{k} \mathbf{x}_{n}+\mathbf{r} \in L([0,1]), \mathbf{k}>0_{L}$ ( $\mathbf{k}$ is a closed subinterval of $] 0,1]$ ). Let $\Sigma$ be the set of admissible permutations of $\mathbf{x}_{1}, \ldots, \mathbf{x}_{n}$ and let $\Sigma^{\prime}$ be the set of admissible permutations of $\mathbf{k x}_{1}+\mathbf{r}, \ldots, \mathbf{k} \mathbf{x}_{n}+\mathbf{r}$. Then, $\Sigma=\Sigma^{\prime}$.

Proof: Let $\sigma \in \Sigma$ and consider some $i, j \in\{1, \ldots, n\}$ with $\mathbf{k x}_{\sigma(i)}+\mathbf{r}<_{L} \mathbf{k} \mathbf{x}_{\sigma(j)}+\mathbf{r}$. Then, it must be $\mathbf{x}_{\sigma(i)}<_{L}$ $\mathbf{x}_{\sigma(j)}$. Since $\sigma \in \Sigma$, this implies $i<j$. Therefore, $\sigma \in \Sigma^{\prime}$. The same reasoning can be done for $\sigma \in \Sigma^{\prime}$, so we have that $\Sigma=\Sigma^{\prime}$. If there is not $i, j$ satisfying $\mathbf{x}_{\sigma(i)}+\mathbf{r}<_{L} \mathbf{k x}_{\sigma(j)}+\mathbf{r}$, then either $\mathbf{x}_{1}=\cdots=\mathbf{x}_{n}$ or $\mathbf{x}_{1}, \ldots, \mathbf{x}_{n}$ form an antichain. In both cases, it is easy to see that $\Sigma=\Sigma^{\prime}$.

Proposition 6: The following items hold:

(i) $\mathbf{C}_{m}^{\text {arith }}\left(\mathbf{k ~ x}_{1}, \ldots, \mathbf{k} \mathbf{x}_{n}\right)=\mathbf{k} \mathbf{C}_{m}^{\text {arith }}\left(\mathbf{x}_{1}, \ldots, \mathbf{x}_{n}\right)$ for every $\mathbf{x}_{1}, \ldots, \mathbf{x}_{n}, \mathbf{k} \in L([0,1])$;

(ii) $\mathbf{C}_{m}^{\text {arith }}\left(\mathbf{x}_{1}+\mathbf{r}, \ldots, \mathbf{x}_{n}+\mathbf{r}\right)=\mathbf{C}_{m}^{\text {arith }}\left(\mathbf{x}_{1}, \ldots, \mathbf{x}_{n}\right)+\mathbf{r}$ for every $\mathbf{x}_{1}, \ldots, \mathbf{x}_{n}, \mathbf{r} \in L([0,1])$ with $\mathbf{x}_{1}+\mathbf{r}, \ldots, \mathbf{x}_{n}+\mathbf{r} \in L([0,1])$.

Proof: By Lemma 1, we know that the set of admissible permutations are the same. The result follows from the fact that the arithmetic mean is stable under any positive linear transformation.

Notice it is not easy to establish an order relation between $\mathbf{C}_{m}^{\text {arith }}$ and other approaches given in Section III. However, since $\mathbf{C}_{m}^{\text {inf }}\left(\mathbf{x}_{1}, \ldots, \mathbf{x}_{n}\right) \leq_{L} \mathbf{C}_{m}^{\text {arith }}\left(\mathbf{x}_{1}, \ldots, \mathbf{x}_{n}\right) \leq_{L}$ $\mathbf{C}_{m}^{s u p}\left(\mathbf{x}_{1}, \ldots, \mathbf{x}_{n}\right)$, we have also the following result.

Proposition 7: For every fuzzy measure $m$ and every $\mathbf{x}_{1}, \ldots, \mathbf{x}_{n} \in L([0,1])$, it holds that

$\mathbf{C}_{m}^{\mathrm{inf}}\left(\mathbf{x}_{1}, \ldots, \mathbf{x}_{n}\right) \leq{ }_{L} \mathbf{C}_{m}\left(\mathbf{x}_{1}, \ldots, \mathbf{x}_{n}\right) \leq_{L} \mathbf{C}_{m}^{\sup }\left(\mathbf{x}_{1}, \ldots, \mathbf{x}_{n}\right)$.

Proof: If we consider the lowerbound, just observe that $\underline{C}_{m}\left(\mathbf{x}_{1}, \ldots, \mathbf{x}_{n}\right)=\underline{C}_{m}^{L e x 1}\left(\mathbf{x}_{1}, \ldots, \mathbf{x}_{n}\right)$. The same reasoning can be done for the upper bounds considering that $\bar{C}_{m}\left(\mathbf{x}_{1}, \ldots, \mathbf{x}_{n}\right)=\bar{C}_{m}^{\text {Lex } 2}\left(\mathbf{x}_{1}, \ldots, \mathbf{x}_{n}\right)$.

Proposition 7 can be easily extended for every admissible order $\preceq_{\mathrm{A}, \mathrm{B}}$, since $\mathbf{C}_{m}^{\text {inf }}\left(\mathbf{x}_{1}, \ldots, \mathbf{x}_{n}\right) \leq_{L}$ $\mathbf{C}_{m}^{\prec \mathrm{A}, \mathrm{B}}\left(\mathbf{x}_{1}, \ldots, \mathbf{x}_{n}\right) \leq_{L} \mathbf{C}_{m}^{\text {sup }}\left(\mathbf{x}_{1}, \ldots, \mathbf{x}_{n}\right)$.

\section{AN APPLICATION OF ADMISSIBLE PERMUTATIONS AND THE INTERVAL-VALUED CHOQUET INTEGRAL IN DECISION MAKING}

In multicriteria decision making problems, the objective is to find the most preferred alternative over a set of $p$ alternatives $A=\left\{a_{1}, \ldots, a_{p}\right\}$ with respect to a set of criteria. We assume that the expert evaluates their preference between each pair of alternatives. Since expressing these preferences with an exact value may be difficult or problematic for he/she, we allow the expert to express the preference of one alternative $a_{i}$ against another $a_{j}$ (with $i, j \in\{1, \ldots, p\}$ ) by means of an interval, i.e. elements of $L([0,1])$. The collection of preferences of every pair of alternatives is done by means of an interval-valued fuzzy relation $\mathbf{R}: A \times A \rightarrow L([0,1])$ (see [26]). The interval $\mathbf{R}\left(a_{i}, a_{j}\right)$ represents the degree to which the alternative $a_{i}$ is preferred to alternative $a_{j}$. For this reason, we also denote $\mathbf{R}$ as an interval-valued fuzzy preference relation or IVFPR.

Once the preference relation is constructed, it must be exploited to obtain a final evaluation of each alternative. To do this, we will consider an interval fusion function $\mathbf{F}_{\mathbf{a}}$ that will by applied to each row of the IVFPR. This operator will aggregate, for each alternative, its preferences, so the higher the value, the more preferred the alternative. Then, for each alternative $a_{i}$, we will obtain a final evaluation $P_{a_{i}}$ which is given by

$$
\begin{array}{r}
P_{a_{i}}=\mathbf{F}_{\mathbf{a}}\left(\mathbf{R}\left(a_{i}, a_{1}\right), \ldots, \mathbf{R}\left(a_{i}, a_{i-1}\right),\right. \\
\left.\mathbf{R}\left(a_{i}, a_{i+1}\right), \ldots, \mathbf{R}\left(a_{i}, a_{p}\right)\right),
\end{array}
$$

where $\mathbf{R}$ is an IVFPR and $\mathbf{F}_{\mathbf{a}}: L([0,1])^{p-1} \rightarrow L([0,1])$ is an interval fusion function.

As we have commented in this paper, the usual partial order of intervals $\leq_{L}$ may not be useful in order to obtain the final decision, i.e. the best alternative or an ordered sequence of alternatives (ordered by preference). This is due to the fact that the interval evaluations obtained $\left(P_{a_{1}}, \ldots, P_{a_{p}}\right)$ may not be comparable. For solving this problem, we propose a simple rule to obtain the best alternative which is based on the set of admissible permutations of $P_{a_{1}}, \ldots, P_{a_{p}}$. For each admissible permutation $\sigma$, each alternative gets the same number of votes as its relative position in the ordered sequence of alternatives (by means of $\sigma$ ). Then, $a_{\sigma(1)}$ will get one vote, $a_{\sigma(2)}$ will get two votes, and so on. After computing every admissible permutation, the best alternative is selected as the one with higher number of votes

The whole decision-making algorithm is summarized in Algorithm 1.

Algorithm 1 Decision Making based on admissible permutations

Require: Set of alternatives $A=\left\{a_{1}, \ldots, a_{p}\right\}$;

IVPFR R : $A \times A \rightarrow L([0,1])$;

Interval fusion function $\mathbf{F}: L([0,1])^{p-1} \rightarrow L([0,1])$.

Ensure: Best alternative $a^{*}$

1: for $i=1, \ldots, p$ do

2: $\quad P_{a_{i}}=\mathbf{F}_{\mathbf{a}_{j=1, j \neq i}^{n}}\left(\mathbf{R}_{C}\left(a_{i}, a_{j}\right)\right)$

3: end for

4: Set votes $V_{a_{1}}, \ldots, V_{a_{p}}=0$

5: Calculate the set of admissible permutations $\sigma_{1}, \ldots, \sigma_{m}$ of $P_{a_{1}}, \ldots, P_{a_{p}}$

6: for $i=1, \ldots, m$ do

7: $\quad$ for $j=1, \ldots, p$ do

8: $\quad V_{a_{\sigma_{i}(j)}}:=V_{a_{\sigma_{i}(j)}}+j$

9: end for

10: end for

11: Assign $a_{*}=\arg \max _{i} V_{a_{i}}$ (if there is a tie, then choose arbitrary one of the tied alternative) 
Example 8: The following example have been taken from [27], where four suppliers $a_{1}, \ldots, a_{4}$ are evaluated taking into account environmental criteria. The expert has provided the following IVFPR:

$$
\left(\begin{array}{cccc}
- & {[0.35,0.45]} & {[0.50,0.70]} & {[0.40,0.50]} \\
{[0.55,0.65]} & - & {[0.60,0.80]} & {[0.20,0.60]} \\
{[0.30,0.50]} & {[0.20,0.40]} & - & {[0.40,0.60]} \\
{[0.50,0.60]} & {[0.40,0.80]} & {[0.40,0.60]} & -
\end{array}\right)
$$

Consider now the following fuzzy measure $m: 2^{\{1,2,3\}} \rightarrow$ $[0,1]$ given by:

$$
\begin{array}{lll}
m(\emptyset)=0 & & \\
m(\{1\})=0.3 & m(\{2\})=0.1 & m(\{3\})=0.35 \\
m(\{1,2\})=0.3 & m(\{1,3\})=0.5 & m(\{2,3\})=0.8 \\
m(\{1,2,3\})=1 & &
\end{array}
$$

Now, we apply $\mathbf{C}_{m}$ to every row of the IVFPR obtaining the following evaluations for each alternative:

$$
\begin{aligned}
& P_{a_{1}}=[0.400,0.510], \\
& P_{a_{2}}=[0.310,0.630], \\
& P_{a_{3}}=[0.285,0.485], \\
& P_{a_{4}}=[0.420,0.620] .
\end{aligned}
$$

Observe that $P_{a_{3}} \leq_{L} P_{a_{i}}$ fori $\in\{1,2,4\}$ and that $P_{a_{1}} \leq_{L}$ $P_{a_{4}}$, but the rest of intervals cannot be compared. This is illustrated in the following Hasse diagram:

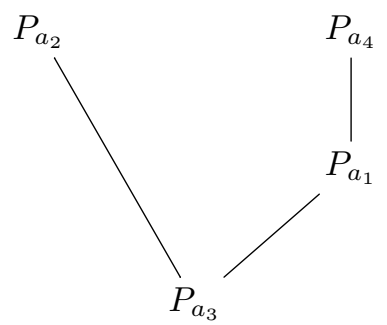

The set of admissible permutations of $P_{a_{1}}, \ldots, P_{a_{4}}$ is composed by $m=3$ permutations, namely $\sigma_{1}, \sigma_{2}, \sigma_{3}$ : $\{1, \ldots, 4\} \rightarrow\{1, \ldots, 4\}$ which are given by:

$$
\begin{aligned}
& \sigma_{1}=(3,1,2,4) \\
& \sigma_{2}=(3,1,4,2) \\
& \sigma_{3}=(3,2,1,4)
\end{aligned}
$$

Finally, let us calculate the votes for each alternative based on $\sigma_{1}, \sigma_{2}, \sigma_{3}$, which are shown in the following table:

\begin{tabular}{c|cccc} 
& $V_{a_{1}}$ & $V_{a_{2}}$ & $V_{a_{3}}$ & $V_{a_{4}}$ \\
\hline$\sigma_{1}$ & 2 & 3 & 1 & 4 \\
$\sigma_{2}$ & 2 & 4 & 1 & 3 \\
$\sigma_{3}$ & 3 & 2 & 1 & 4 \\
\hline & 7 & 9 & 3 & 11
\end{tabular}

This means that $V_{a_{1}}=7, V_{a_{2}}=9, V_{a_{3}}=3$ and $V_{a_{4}}=11$, so we can order the alternatives with the following preference

$$
a_{3} \prec a_{1} \prec a_{2} \prec a_{4} .
$$

\section{CONCLUSIONS}

In this work we have proposed a new fusion mechanism for interval data based on the Choquet integral that considers the ordinal structure of data in the fusion process. Although there exist some other approaches to the integration of inter$\mathrm{val} /$ intuitionistic data (see for example [28], [29], [30], [31], [32], [33], [34], [35] for some examples in the intuitionistic framework), they do not extend the standard Choquet integral. That is, when we consider real numbers, we expect these extensions to recover the result of the original Choquet integral of real inputs.

Our approach, that recovers the original definition when considering real numbers, is based on a consensus (or agreement) between interval-valued Choquet integrals constructed with different settings. In order to do so, we have first proposed the concept of an admissible permutation. This has allowed to calculate the different number of arrangements (satisfying some basic properties) of the input data. Then, the result of each interval-valued Choquet integral based on a specific permutation is finally fused into a single interval output that models the consensus among the different permutations.

The proposed fusion procedure focuses on the Choquet integral. However, it is easy to see that it can be extended to those fusion functions based on the arrangement of data, such as the OWA operators or the Sugeno integral. Moreover, we think that it would be interesting to study the importance of each individual permutation, allowing therefore to calculate the consensus by means of weighted functions or some other functions that simulate the attitude of the final user.

\section{ACKNOWLEDGMENT}

This work has been partially supported by MINECO, AEI/FEDER,UE under grant TIN2016-77356-P, by grant VEGA 1/0420/15, by grant LQ1602 IT4Innovations excellence in science and by the Research Services of the Universidad Publica de Navarra.

\section{REFERENCES}

[1] G. Beliakov, A. Pradera, and T. Calvo, Aggregation Functions: A Guide for Practitioners. Springer: Studies in Fuzziness and Soft Computing 221, 2007.

[2] G. Beliakov, H. Bustince, and T. Calvo, A Practical guide to Averaging Functions. Springer: Studies in Fuzziness and Soft Computing 329, 2016.

[3] M. Grabisch, J.-L. Marichal, R. Mesiar, and E. Pap, Aggregation Functions. Cambridge University Press, 2009.

[4] B. Khaleghio, A. Khamis, F. O. Karray, and S. N. Razavi, "Multisensor data fusion: A review of the state-of-the-art," Information Fusion, vol. 14, pp. 28-44, 2013.

[5] D. Paternain, J. Fernandez, H. Bustince, R. Mesiar, and G. Beliakov, "Construction of image reduction operators using averaging aggregation functions," Fuzzy Sets and Systems, vol. 261, pp. 87-111, 2015.

[6] D. Paternain, M. J. Campión, R. Mesiar, I. Perfilieva, and H. Bustince, "Internal fusion functions," IEEE Transactions on Fuzzy Systems, vol. 26, pp. 487-503, 2018.

[7] K. Atanassov, "Intuitionistic fuzzy sets," Fuzzy Sets and Systems, vol. 20, pp. 87-96, 1986.

[8] H. Bustince, "Interval-valued fuzzy sets in soft computing," International Journal of Computational Intelligent Systems, vol. 3, pp. 215-222, 2010.

[9] B. Bedregal, G. Beliakov, H. Bustince, T. Calvo, R. Mesiar, and D. Paternain, "A class of fuzzy multisets with a fixed number of memberships," Information Sciences, vol. 189, pp. 1-17, 2012. 
[10] H. Bustince, E. Barrenechea, M. Pagola, J. Fernandez, Z. Xu, B. Bedregal, J. Montero, H. Hagras, F. Herrera, and B. D. Baets, "A historical account of types of fuzzy sets and their relationships," IEEE Transactions on Fuzzy Systems, vol. 24, pp. 179-194, 2016.

[11] E. Barrenechea, H. Bustince, B. D. Baets, and C. Lopez-Molina, "Construction of interval-valued fuzzy relations with application to the generation of fuzzy edge images," IEEE Transactions on Fuzzy Systems, vol. 19, pp. 819-830, 2011.

[12] A. Jurio, M. Pagola, R. Mesiar, G. Beliakov, and H. Bustince, "Image magnification using interval information," IEEE Transactions on Image Processing, vol. 20, pp. 3112-3123, 2011.

[13] R. J. Aumann, "Integrals of set-valued functions," Journal of Mathematical Analysis and Applications, vol. 12, pp. 1-12, 1965.

[14] H. Bustince, M. Galar, B. Bedregal, A. Kolesárová, R. Mesiar, and A. New, "Approach to interval-valued choquet integrals and the problem of ordering in interval-valued fuzzy set applications," IEEE Transactions on Fuzzy Systems, vol. 21, pp. 1150-1162, 2013.

[15] G. Choquet, "Theory of capacities," Annales de l'Institute Fourier, vol. 5, pp. 131-292, 1954.

[16] M. Komorníková and R. Mesiar, "Aggregation functions on bounded partially ordered sets and their classification," Fuzzy Sets and Systems, vol. 175, pp. 48-56, 2011.

[17] G. Deschrijver, C. Cornelis, and E. E. Kerre, "On the representation of intuitionistic fuzzy t-norms and t-conorms," IEEE Transactions on Fuzzy Systems, vol. 12, pp. 45-61, 2004.

[18] H. Bustince, J. Fernandez, A. Kolesárová, and R. Mesiar, "Generation of linear orders for intervals by means of aggregation functions," Fuzzy Sets and Systems, vol. 220, pp. 69-77, 2013.

[19] Z. S. Xu and R. R. Yager, "Some geometric aggregation operators based on intuitionistic fuzzy sets," International Journal of General Systems, vol. 35, pp. 417-433, 2006.

[20] V. Torra, Y. Narukawa, and M. Sugeno, eds., Non-Additive Measures, Theory and Applications (Studies in Fuzziness and Soft Computing Series 310), Springer. 2014.

[21] D. Paternain, H. Bustince, M. Pagola, P. Sussner, A. Kolesárová, and R. Mesiar, "Capacities and overlap indexes with an application in fuzzy rule-based classification systems," Fuzzy Sets and Systems, vol. 305, pp. 70-94, 2016.

[22] R. Mesiar and A. Stupňanová, "Decomposition integrals," International Journal of Approximate Reasoning, vol. 54, pp. 1252-1259, 2013.

[23] Y. Even and E. Lehrer, "Decomposition-integral: unifying choquet and the concave integrals," Economic Theory, vol. 56, pp. 33-58, 2014.

[24] T. Wilkin and G. Beliakov, "Weakly monotonic averaging functions," International Journal of Intelligent Systems, vol. 30, pp. 144-169, 2015.

[25] H. Bustince, J. Fernandez, A. Kolesárová, and R. Mesiar, "Directional monotonicity of fusion functions," European Journal of Operational Research, vol. 244, pp. 300-308, 2015.

[26] H. Bustince and P. Burillo, "Mathematical analysis of interval-valued fuzzy relations: Application to approximate reasoning," Fuzzy Sets and Systems, vol. 113, pp. 205-219, 2000.

[27] J. Wu, F. Chiclana, and H. Liao, "Isomorphic multiplicative transitivity for intuitionistic and interval-valued fuzzy preference relations and its application in deriving their priority vectors," IEEE Transactions on Fuzzy Systems, vol. 26, pp. 193-202, 2018.

[28] J. Ashayeri, G. Tuzkaya, and U. R. Tuzkaya, "Supply chain partners and configuration selection: An intuitionistic fuzzy choquet integral operators based approach," Expert Systems with Applications, vol. 39, pp. 36423649, 2012.

[29] W. J. Liu, Z. Z. Lin, F. S. Wen, and G. Ledwich, "Intuitionistic fuzzy choquet integral operator-based approach for black-start decisionmaking," IET Generation, Transmission \& Distribution, vol. 6, pp. 378386, 2012.

[30] C. Tan and X. Chen, "Intuitionistic fuzzy choquet integral operator for multi-criteria decision making," Expert Systems with Applications, vol. 37, pp. 149-157, 2010.

[31] C. Tan and X. Chen, "Induced intuitionistic fuzzy choquet integral operator for multicriteria decision making," International Journal of Intelligent Systems, vol. 26, pp. 659-686, 2011.

[32] G. Tuzkaya, "An intuitionistic fuzzy choquet integral operator based methodology for environmental criteria integrated supplier evaluation process," International Journal of Environmental Science and Technology, vol. 10, pp. 423-432, 2013.

[33] J. Wu, F. Chen, C. Nie, and Q. Zhang, "Intuitionistic fuzzy-valued choquet integral and its application in multicriteria decision making," Information Sciences, vol. 222, pp. 509-527, 2013.

[34] Z. S. Xu, "Choquet integrals of weighted intuitionistic fuzzy information," Information Sciences, vol. 180, pp. 726-736, 2010.
[35] X. Zhang, Z. Xu, X. Yu, S. Value, and C. Integral-based, "Operators for aggregationg correlated intuitionistic fuzzy information," Information, vol. 14, pp. 1847-1857, 2011.

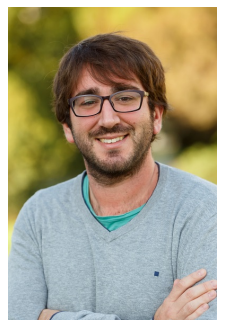

Daniel Paternain received the M.Sc. and Ph.D. degrees in Computer Science from the Public University of Navarra, Pamplona, Spain, in 2008 and 2013, respectively. He is currently Lecturer with the Department of Statistics, Computer Science and Mathematics, Public University of Navarra. He is the author and co-author of almost 25 paper in JCR and more than 50 international conference communications. His research interests include both theoretical and applied aspects of aggregation functions, image processing and machine learning. His main contributions are based on the use of aggregation techniques and new information fusion procedures, such as aggregation functions, penalty functions or fuzzy integrals in image processing and supervised classification algorithms.

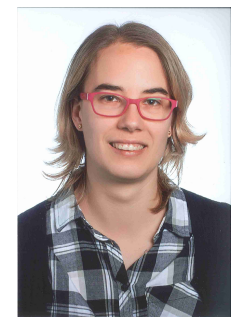

Laura De Miguel Laura De Miguel received degree in Mathematics from the University of Zaragoza, Spain, in 2012 and the Ph.D. degree in Computer Sciences from the Public University of Navarra, Pamplona, Spain, 2017. She is currently an Assistant Professor in the Department of Statistics, Computer Science and Mathematics at the Public University of Navarra. Her research interests are fuzzy sets and their generalizations, aggregation functions and fuzzy measures.

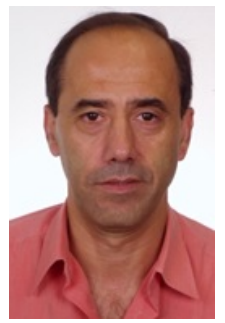

Gustavo Ochoa Gustavo Ochoa received the M.Sc. and Ph.D. degrees in mathematics from the University of Zaragoza, Spain, respectively in 1981 and 1984. He is currently an Assistane Lecturer with the Department of Mathematics, Public University of Navarre, Pamplona, Spain. His research interests include group theory, representations theory and more reccently fuzzy sets and aggregation functions applied to decisión making and image processing.

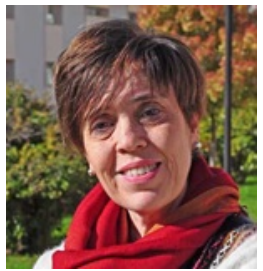

Inmaculada Lizasoain Inmaculada Lizasoain received the Ph.D. degree in mathematics from the Public University of Navarre, Spain, in 1995, where he is currently an Associate Professor with the Department of Statistics, Computer Sciences and Mathematics and INAMAT Research Institure. Her research interests, that include representation group theory, aggregation functions of lattice fuzzy sets and their applications to algebraic automata, decision making and image processing, have given place to more than 20 research papers. 


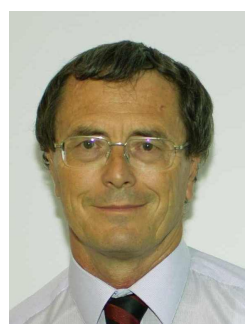

Radko Mesiar received the Graduate degree in mathematics from the Faculty of Mathematics and Physics and the Ph.D. degree with the thesis "Subadditive martingale processes" from Comenius University, Bratislava, Slovakia, in 1974 and 1979, respectively. He received the D.Sc. degree from the Academy of Sciences of the Czech Republic, Prague, Czech Republic, in 1996. Since 1978, he has been a Member of the Faculty of Civil Engineering, Department of Mathematics, Slovak University of Technology, Bratislava, where he is currently a Full Professor. In 1983, he became an Associate Professor and, in 1998, a Professor. In 1995, he became a Fellow Member of the Czech Academy of Sciences, Institute of Information and Automation, Praha, Czech Republic, and of IRAFM, University of Ostrava, Ostrava, Czech Republic, in 2006. He is the coauthor of two scientific monographs: Triangular Norms (Norwell, MA, USA: Kluwer, 2000) and Aggregation Functions (Cambridge, U.K.: Cambridge Univ. Press, 2009), and five edited volumes. He is the author of more than 250 papers. His research interests include measure and integral theory, uncertainty modelling, fuzzy sets and fuzzy logic, multicriteria decision support, copulas, triangular norms, aggregation operators and intelligent computing. $\mathrm{He}$ has been a Fellow of the International Fuzzy Systems Association since 2011. $\mathrm{He}$ is a Founder and Organizer of the FSTA, ISCAMI, ABLAT, and AGOP conferences.

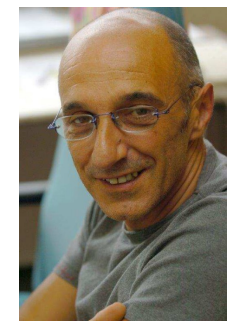

Humberto Bustince is full professor of Computer Science and Artificial Intelligence in the Public University of Navarra. He is the main researcher of the Artificial Intelligence and Approximate Reasoning group of this University, whose main research lines are both theoretical (aggregation functions, information and comparison measures, fuzzy sets and extensions) and applied (image processing, classification, machine learning, data mining and big data). He has authored more than 200 works, according to Web of Science, in conferences and international journals, with around 100 of them in journals of the first quartile of JCR. Moreover, five of these works are also among the highly cited papers of the last ten years, according to Science Essential Indicators of Web of Science. He is editor-in-chief of the online magazine Mathware \& Soft Computing of the European Society for Fuzzy Logic and Technologies (EUSFLAT) and of the Axioms journal. He is associated editor of the IEEE Transactions on Fuzzy Systems journal and member of the editorial board of the journals Fuzzy Sets and Systems, Information Fusion, International Journal of Computational Intelligence Systems and Journal of Intelligent \& Fuzzy Systems. He is coauthor of a monography about averaging functions and co-editor of several books. He has organized some re-known international conferences such as EUROFUSE 2009 and AGOP 2013. He is Senior member of the IEEE Association and Fellow of the International Fuzzy Systems Association (IFSA). 